\title{
ROBOTIC ASSISTED DEEP BRAIN STIMULATION NEUROSURGERY: FIRST STEPS ON SYSTEM DEVELOPMENT
}

\author{
Carlos Faria $^{1 *}$, Estela Bicho ${ }^{1}$, Manuel Rito ${ }^{3}$, Luís Louro $^{1}$, Sérgio Monteiro ${ }^{1}$ and Wolfram Erlhagen ${ }^{2}$ \\ ${ }^{1}$ Department of Industrial Electronics and Center Algoritmi, University of Minho, Portugal \\ ${ }^{2}$ Department of Mathematics and Applications and Center of Mathematics, University of Minho, Portugal \\ ${ }^{3}$ Service of Neurosurgery, Coimbra University Hospital, Portugal \\ \{cfaria, estela.bicho, 1.louro, sergio\}@dei.uminho.pt; manuelantoniorito@gmail.com; wolfram.erlhagen@math.uminho.pt
}

\begin{abstract}
The advantages of Deep Brain Stimulation (DBS) lead to an increasing number of stereotactic DBS surgeries, which are extensive procedures that require extreme precision and steadiness of tool handling. Robotic manipulators known for their consistency, movement precision and steadiness have the potential to be remarkable tools to assist the neurosurgeons and can refine the quality/working conditions, while improving surgery outcome. Currently, robotic systems for stereotactic neurosurgeries with simple/pragmatic low budget solutions that fulfil the surgeons' needs are not yet available. Thus, we have been asked to develop such robotic system. In this paper we present our first steps toward such endeavour. Specifically, we implemented a simulation environment for robotic assisted DBS neurosurgery that allows emulating several hardware setups within the operating room, and to test and assess their performance. The simulator is useful not only as tool for developing specialized control applications, but also for training clinicians. First results support the viability of the sought solution and open way to future developments.
\end{abstract}

\section{KEY WORDS}

Robotic neurosurgery; Stereotactic electrode implantation; DBS

\section{Introduction}

Deep Brain Stimulation (DBS) is a surgical treatment that consists in the stereotactic implantation of several electrodes connected to implanted pulse generators (IPG), responsible to deliver controlled electrical stimuli to specific regions of the basal ganglia [1]. DBS is currently established as one of the best treatments to relieve symptomatic expression of several major neurological disorders, and unlike the alternative drug-based or ablative solutions it is non-destructive and reversible [2][3]. It is currently indicated for symptomatic treatment of Parkinson's disease (PD), dystonia, essential tremor and parkinsonian tremor, epilepsy, neurological pain and psychiatric disorders [3]. Each disorder has a significant impact in society, due to the level of impairment associated and/or to the numbers of afflicted individuals [4][5][6].
The growing acceptance and overall success of DBS, is being reflected on the increasing number of patient's eligible, of neurosurgery services adopting this technique and of prescribed DBS surgeries [7]. This surgery is physically and psychologically demanding for the medical team since it drags out for long hours, during which it is required extreme tool handling precision and steadiness. The combination of surgery characteristics with the growing search for DBS treatment, has pushed neurosurgery services to the limits of their capability to perform the required number of DBS surgeries. Consequently, neurologists need to prioritize patients and deprive others from the treatment because the healthcare providers offer cannot match the search.

The benefits of DBS therapy and the limitations that persist in the current approach, lead medical teams to improve their methodologies and motivated engineers to develop new surgical tools, which will eventually ameliorate the overall success of the procedure. The dissemination and progress of technology, brought robotic systems to precision and repeatability standards that far exceed the human capabilities [8]. More industries types are currently embracing this technology for their unmatched potential, which lead robotic manufacturers to continuously launch better systems for rather affordable prizes. The robotic manipulators are nowadays so accessible that after some market search and inquiring, it was found that a robotic system with a repeatability below $0.01 \mathrm{~mm}$, costs roughly half the price of the mechanical frame actuated through mechanical screws used in stereotactic procedures solely to position the electrodes in the desired trajectory towards the target.

The advantages from robotic neurosurgery and DBS treatment are just too inviting, and motivated our team to study and draft a solution that could profit from merging both. After understanding the potential behind such hypothesis, we sought potential systems oriented for surgeries that could fill this need. With no discredit for the solutions currently commercially available and according to the opinion of actual users, the robotic solutions are just adapted systems or exceedingly expensive, bereft of a pragmatic sense and eventually pile superfluous features that deviate from their main goal and just add up to the final price.

Our work aims to develop a simple, easy to use and 
economically affordable robotic system to assist the neurosurgeon during several tasks performed within the operating room in a DBS procedure. Along this paper, several fundamental issues are addressed like: How can a robotic manipulator be of use within the operating room? How should it fit the target environment? What robotic systems are better suited for our purpose? Can we devise a manipulation algorithm according to the expected performance? Can we devise a simulation environment that allows the developers of the robotic system to test and to anticipate possible implementation issues? And that holds the potential to serve as a tool for training clinicians?

The organization of this paper is as follows: section 2 describes a standard DBS procedure and discusses the utility of a robotic manipulator in DBS surgery. In section 3 is presented a state of the art study about neurosurgical robots adaptable or oriented to DBS surgery and selected industrial robotic systems for our project. Section 4 , describes several components of the devised simulation environment. The paper ends in section 5 with conclusion and future guidelines.

\section{Deep Brain Stimulation}

One of our primary tasks is to briefly describe the key steps of a stereotactic DBS surgery and to figure out how can a robotic manipulator be used. To acquire practical insight, we attended to a DBS neurosurgery that took place in the Service of Neurosurgery in the Coimbra University Hospitals in Portugal and was conducted in a patient affected by Parkinson's disease. Additional information regarding DBS surgery can be found at [3][9][10][11].

\subsection{Surgery}

Upon being considered eligible for DBS surgery, the patient is subjected to several imaging exams previous and after fixating the reference system of the stereotaximeter to the skull. The MRI and CT scans collected are introduced in a imaging software responsible for matching both and creating a $3 \mathrm{D}$ representation of the patient skull and neurological structures. The neurosurgeon's team then pinpointed the target locations, from which it was outlined the better trajectory for electrode insertion, which should always avoid key and vascular structures.

Intraoperatively, the stereotactic frame used to guide the electrodes into the patient's head is first calibrated and tested using a phantom device, (figure 1). The frame positions a stiff and thin metal rod whose tip should meet the target coordinates simulated by the phantom device. Both the phantom as the stereotactic frame coordinates are set through mechanical screws, adjusted according to millimetric scales engraved between moving components. If both tips coincide, the trajectory directives are correct and the stereotactic frame is dismounted from the phantom device and mounted in the reference system of the stereo- taximeter attached to the patient's head.

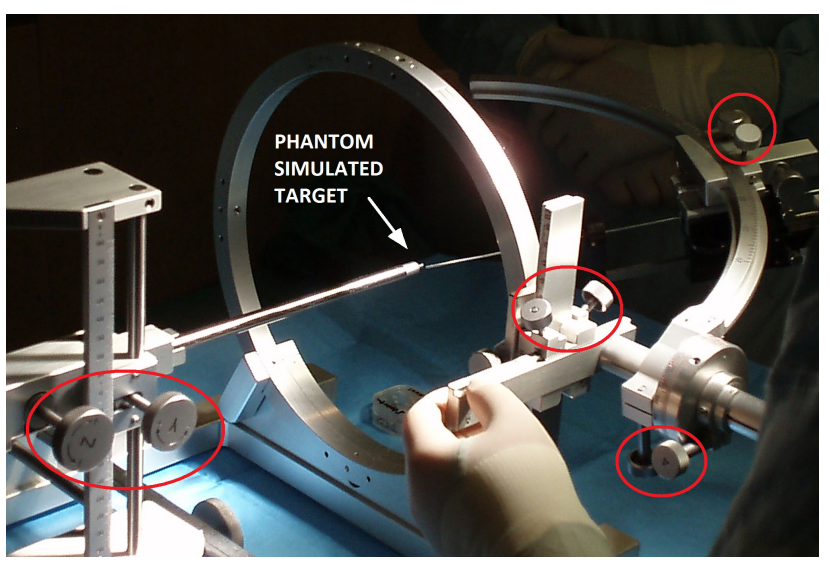

Figure 1. Verification of stereotactic frame coordinates using a phantom device. Mechanical screws marked in red.

Then the metal rod used to confirm the target coordinates in the phantom, was positioned along the desired trajectory to mark the entry position for the electrode lead's. The stereotactic frame was moved away to clear the neurosurgeon's line of sight and workspace so he could proceed to make the scalp incision, and drill a burr hole to provide access to the intracranial cavity. The frame is once again moved to its assigned position along with a driver mechanism responsible for lowering each electrode lead's collinear to the planned trajectory.

In a DBS procedure it is used different sets of electrodes, being the first set of microelectrodes for recording cortical electrical signals. In the attended surgery, the neurosurgeons placed 5 electrodes arranged in a cross fashion, to cover a broader area of cortical tissue. The electrodes were moved downward until $15 \mathrm{~mm}$ from the target pinpointed position. The patient's sedation varied along the procedure and at this point it was slightly reduced in order to assess the cortical functioning in an awake state. The anesthesia however, must not be over reduced in order to avoid further pain and stress to the patient. From here, the team of neurologists collected and evaluated each signal iteratively at each millimeter until $5 \mathrm{~mm}$ from the target. Henceforward, the electrodes advanced half by half millimeter until the target position and past it. This direct mapping of electrical activity was then studied to find the most proximal location to the sensorimotor region inside the nucleus, and such information is either used to confirm or to adjust the initial coordinates obtained from the imaging software.

The reading microelectrodes were then replaced for stimulating micro/macroelectrodes, which were inserted in the positions where the cortical electrical signals gave better feedback. From this point neurologists followed an iterative methodology where they changed the depth of electrodes or adjusted the stimulating signal amplitude, and then evaluated how the patient's symptoms progressed. 
Upon finding the ideal placement and stimulation signal properties, the micro/macroelectrodes were replaced for a definitive quadripolar macro electrode that would later be connected to an IPG device (figure 2).

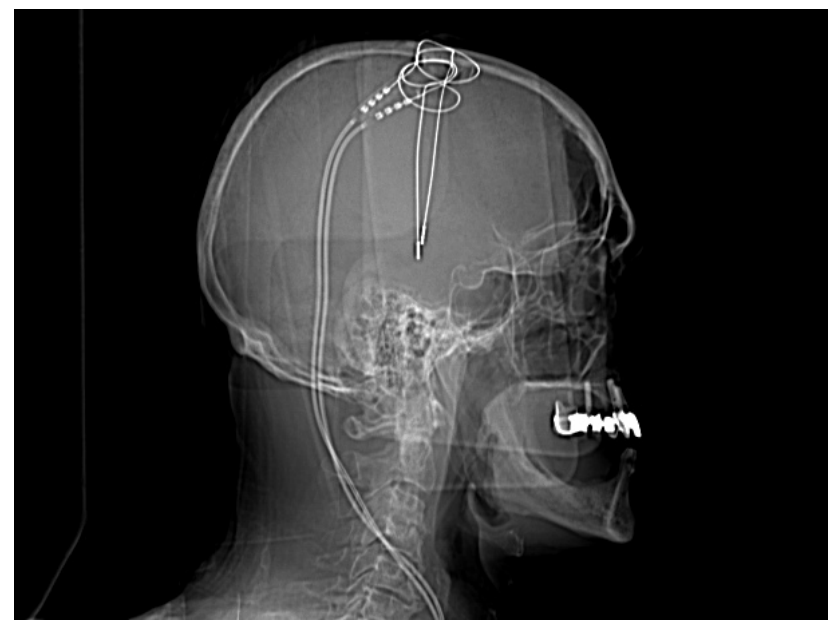

Figure 2. Post operative X-ray showing the placement of bilateral quadripolar macroelectrodes.

If the surgery is bilateral or several targets are to be covered, the procedure should be throughout repeated, starting again from the phantom device coordinate testing stage. DBS surgeries can drag for long periods of time, the one here described lasted for approximately 18 hours.

\subsection{Robotic manipulator utility}

Upon acquiring practical insight relative to DBS surgery and brainstorming with neurosurgical partners, we were able to answer some pending questions about the ultimate goal of introducing a robotic manipulator and to understand what was expected from such tool. To start, DBS surgery is usually a time demanding procedure through which the surgical team must remain utterly focused because any trajectory error or undesired tool displacements can imply great risk to the patient. Robotic manipulators known for their untiring steadiness and movement precision could be a significant help to surgeons, both to improve their working conditions by providing consistent tool positioning and electrode guiding and also by possibly ameliorating the surgery outcome. According to the data collected and to the surgery prerequisites in general, the robotic system would have a positive impact inside de operating room by:

1. Allowing the trajectory information to be managed by hardware, using a communication protocol that would link the imaging software to the robotic controller.

2. Avoiding the use of mechanical devices controlled through mechanical screws, whose precision is limited by the human vision resolution and movement dexterity, and are susceptible to clearance or calibration errors.
3. Avoiding the tiring and potential error inducing process of mount/dismount of the stereotactic frame for each set of target coordinates.

4. Positioning itself at the desired trajectory and change its end-effectors according to the task without moving from the planned position.

5. Providing assistance to young and less experienced neurosurgeons as an assistive and training platform and enabling senior highly experienced neurosurgeons, who might lost some dexterity, to continue performing surgeries later in their careers.

6. Opening the possibility for frameless stereotactic surgery.

\section{Robots for stereotactic DBS}

A state of the art study on robotic systems build for neurosurgery, either commercially available or at research stage, that were oriented or could potentially be adapted to stereotactic surgeries, was conducted. The information about each system's main features will be summarily displayed and it will be given some additional focus to potential drawbacks. The robotic projects oriented to stereotactic procedures, included in this state of the art were tracked down from MeRoDa (Medical Robotic Database) ${ }^{1}$, by definition a database that compiles all the robotic projects oriented to medical applications, and several review papers about robotic neurosurgery [12][13][14][15].

From all the projects we narrowed our scope to eight: three robotic systems adaptable to stereotactic procedures and five oriented solutions.

\subsection{Adaptable robotic systems}

Starting with the adaptable solutions we reviewed the Evolutionl from Universal Robot Systems [16], the Minerva from the Microengineering laboratory in the Swiss Federal Institute of Technology [17] and NeuroArm from the University of Carlgary [18]. Only the Evolutionl is currently available at market and to the extent of our knowledge the Minerva project was discontinued.

Evolution1 is a 4 DOF (degrees of freedom) hexapod robot based on a parallel actuator, designed for extreme precision handling of endoscopic applications for brain or spine surgeries, which could eventually be adapted to handle other types of instrumentation. However, the large payload capacity provided by the parallel architecture is superfluous as DBS instrumentation weight is not restrictive and the limited workspace requires that the robot is previously positioned very close to the surgical field, thus subtracting the neurosurgeon's workspace.

The Minerva system was a 5 DOF robot with 6 actuated joints used for instrumentation guiding within a CT

\footnotetext{
${ }^{1}$ Available at: http: //www. umm. uni-heidelberg.de/ apps/ortho/meroda/
} 
scanner machine. It aimed to combine the advantages of a robotic tool with the dynamic tracking of instrumentation directly from tomography scans. Although promising, the project solution wasn't able to overcome some fundamental drawbacks as it still relied in a reference stereotactic frame, it didn't have an acceptable movement flexibility and the surgeries were longer with few advantages to support it.

The NeuroArm awarded project aims for a MRcompatible tele operated robot formed by two 7 DOF manipulators, thus enabling neurosurgeon's to perform microsurgery or stereotaxy with an online feedback of the instrumentation. It also brings top-notch features like: online 3D image reconstruction; haptic feedback on robotic manipulation and hand controller revolutionary design. Despite the remarkable characteristics and front end development, there is still doubt concerning the actual need of online MRI in light of stereotactic DBS surgery. Being all the actions pre-coordinated and strictly performed, the information retrieved from MRI scans does rarely justify the exorbitant costs associated to this technology.

\subsection{Oriented robotic systems}

Now for the projects more suited to the DBS stereotactic procedures we selected, Neurobot from the Imperial College of Science, Technology and Medicine in London [19], NeuroMate from Renishaw ${ }^{\circledR}$ [20], Pathfinder from Prosurgics Ltd. [21], Robocast from Neuroengineering and medical robotics Laboratory of the Politecnico di Milano [22] and Rosa from Medtech ${ }^{\mathrm{TM}}$ [23]. Only Neurobot and Robocast are still in development/research stage, all the others are commercially available.

Neurobot system consists of a 4 DOF robotic arm and a simulator image guided system. The surgical plan is based in preoperative MRI data sets, then the neurosurgery is performed through the robot, which is controlled by the surgeon. The surgeon is able to manipulate the orientation coordinates (Yaw, Pitch and Endoscope rotation) and the depth of the instrumentation. This robotic system main disadvantages include the need to previously move and register the robot's position near the entry point before proceeding with the surgery and the reduced flexibility.

The NeuroMate system is a 5 DOF image guided serial manipulator built to assist neurosurgeons in supporting and stabilizing tools in stereotactic procedures. It has an embedded state of the art planning and visualization system that supports CT, MRI and angiographic images. This system presents a variety of features that we crave for in our solution however, the static robotic base compromises its mobility, making it unwieldy and the integrated imaging system forces the neurosurgery service to adapt and operate with this new software disregarding the current one being used.

The Pathfinder system composed by a 6 DOF serial manipulator mounted in a mobile and stable platform, with an attaching mechanism that binds the platform to the stereotactic reference system also fixated to the pa- tient's head, which guarantees that the transformation from the robot base to the patient's surgical referential is kept. This transformation is computed by registering the position of several fiducial markers attached to the patient's skull or skin. The robot platform is positioned sideward from the patient, to avoid obstructing the neurosurgeon's view or workspace. The most common issues with Pathfinder are related to undetected fiducial markers or displacements caused by skin movements.

Robocast is based in a multi-robotic 13 DOF architecture composed by a parallel, serial and linear actuators for extreme precision probe alignment in keyhole neurosurgery. The first actuator is the serial robot Pathfinder known as the gross positioner, which is connected in series to the fine positioner a $6 \mathrm{DOF}$ parallel robot that in its turn is linked to a linear actuator responsible for lowering the probes in a linear trajectory. The only system's foresaw downside is the theoretically increased probability of failure due to the serial disposition of several complex components.

The recent and publicized Rosa system includes a 6 DOF robotic manipulator and a neurosurgical image registration and visualization software. Built towards precise targeting and dexterous handling of minimally invasive instrumentation, Rosa has a mobile platform and a position recognition method based in laser telemeters and fiducial markers. This solution main flaws seem to be the acquisition and maintenance costs, and the mandatory integrated imaging software.

\subsection{Industrial robotic systems}

The characteristics and guidelines followed in DBS surgery establish a set of requirements and conditions, for the typology and maneuverability of the selected robotic system to assist the surgeon. Concerning the robotic systems available to industry, we summed up key robotic features in order to select the better fitted systems.

Firstly the choice of serial manipulators over a parallel mechanism, was based in the need of movement flexibility and a broader workspace to better suit the space available without obstructing the surgeon. Parallel actuators have significantly smaller workspaces and excel at precise manipulation of larger payloads, which falls outside the scope of our problem.

The ideal number of DOF is a complicated question to answer. It defines the number of independent motions performed by the manipulator. To reach any $3 \mathrm{D}$ position in space with any specific orientation within the dexterous workspace the robot needs at least 6 DOF. Increasing the number of DOF above 6 will create redundancy, which enables the manipulator to reach the same coordinates with different configurations. The controller can now choose the best approach to avoid collisions with people or equipment; however, additional joints also add to end-effector position and orientation errors since the robot structure is serial.

In terms of joint actuators, stereotactic surgical pro- 
cedures demand low speed/inertia, high precision and rigid motions. Neither execution time nor payload are restrictive task variables, instead it is expected from the robotic system to behave in a low velocity profile for safety concerns and incorporate stiff joint motion to guarantee steady positioning and handling of instrumentation in a desired trajectory.

We gathered information from the major renown and market established companies like: Abb, Adept, Epson, Fanuc, Kuka, Mitsubishi, Motoman, Nachi, Schunk, Staubli, Toshiba and Universal Robots. From the wide offer of products, we restricted our search to 6 DOF anthropomorphic serial manipulators whose robotic and controller weights should fall under $40 \sim 50 \mathrm{~kg}$ to avoid compromising the system's portability. For the robot to assist the neurosurgeon's in drilling tasks, which involve a certain level of force, we discourage using systems whose payload capacity are less than $3 \mathrm{~kg}$. Finally, to assure consistent positioning, we established as an acceptable repeatability value, $0.1 \mathrm{~mm}$. The most differentiating and important feature "accuracy" was left unattended in the selection process because most companies don't share such information. In the end, the most promising systems were the $A b b I R B$ 120 and Motoman MH5. It was also included the Schunk Amtec LightWeightArm-II anthropomorphic arm, since it is available for testing in our laboratory and also to study the behavior of a 7 DOF manipulator with elbow redundancy.

\section{Developed Solution}

Upon selecting the robotic systems and obtaining information about their dimensions, workspace, joint typology, position and limits, we started developing the control algorithms. Any robotic task can be divided into a sequence of elemental motions either point to point or based in velocity/acceleration profiles. The serial manipulator is controlled by the displacement of each joint along its kinematic chain. To move the end-effector from point A to point B, there must be a coordinated action from each joint. Robotic kinematics studies the geometry of motion of a robotic structure, relative to a coordinated system and disregarding any forces or moments involved.

\subsection{Kinematic problem}

Each joint displacement was described in the Joint space by a $(n \times 1)$ joint vector,

$$
\mathbf{q}=\left[\begin{array}{lll}
q_{1} & \ldots & q_{n}
\end{array}\right]^{T}
$$

The Cartesian space describes the spatial coordinates and orientation of the robot's end-effector $\left(\mathbf{x}_{\mathbf{e}}\right)$ and can be represented by a XYZ $(3 \times 1)$ vector for position $\mathbf{p}_{\mathbf{e}}$ and another $(3 \times 1)$ vector for orientation $\gamma_{\mathbf{e}}$, according to the Euler Angles.

$$
\mathbf{x}_{\mathbf{e}}=\left[\begin{array}{c}
\mathbf{p}_{\mathbf{e}} \\
\gamma_{\mathbf{e}}
\end{array}\right]
$$

The computed Geometric Kinematics expresses the relationship between Joint and Cartesian space. The forward kinematics,

$$
\mathbf{x}_{\mathbf{e}}=f(\mathbf{q})
$$

provide the end-effector position and orientation given the joint displacements; they were computed following the Denavit-Hartenberg convention. On the other hand, the inverse kinematics,

$$
\mathbf{q}=f^{-1}\left(\mathbf{x}_{\mathbf{e}}\right)
$$

return the joint angles for the manipulator to reach a feed in position and orientation, and were calculated using analytic/geometric hybrid method. Unlike forward kinematics it doesn't always have a possible and determined solution for the given variables, due to the highly non linearity of equations.

Differential kinematics establish the relations between the Joint and Cartesian space velocities. The Differential Direct Kinematics compute the tool velocities from each joint velocity,

$$
\mathbf{v}_{\mathbf{e}}=g(\dot{\mathbf{q}})
$$

and from the inverse relation, one can calculate each joint velocity to achieve a final end-effector linear and angular velocity through Differential Inverse Kinematics,

$$
\dot{\mathbf{q}}=g^{-1}\left(\mathbf{v}_{\mathbf{e}}\right)
$$

The problem formulation is similar to geometric kinematics, but instead of dealing with absolute positions, the joint and end-effector variables are relative to velocities or displacements. Differential kinematics, besides providing a velocity-based control over the manipulator also allow the user to have feedback on joint velocities/torques, to control the arm based on incremental displacements and to dynamically constraint joints during back-driving movements [24]. Both features are useful to assist a neurosurgeon in DBS surgery since the depth of electrodes must be controlled along the planned trajectory only, and a constraint back-driving feature would abet the neurosurgeon by restraining any action outside the surgical field.

Due to space limitations, it wasn't possible to describe the kinematic expressions for each manipulator. For complete information about this matter please consult [25].

\subsection{Simulator}

The selected manipulators and the correspondent kinematics were tested in a simulation environment. Before acquiring any robotic equipment due to the costs involved, it is essential to test the solution for potential issues. Besides, the simulator is also an useful tool to debug the control application and to train neurosurgeons with the control application user interface, which manipulates the robot. 
Under the outlined objectives for our system, it was defined a set of required obligatory features for the simulator. In first place it is expected of the simulator to represent as close as possible each robotic system not only concerning its graphical representation and size but also regarding its physical counterpart. Secondly to assess the performance of the robotic manipulator within the operating room, the virtual world simulated should resemble the working environment.

Despite the variety of free robotic simulator softwares like Player-Stage-Gazebo, USARSim, SimRobot or paid solutions such as Microsoft Robotics Developer Studio and Webots we chose to implement our own robotic software. The CoopDynSim 3D robotics simulator gathers several inviting features like the flexibility to include custom components and objects, a modular architecture based on a client-server topology using middleware abstraction layer for communication [26]. The socket base interface allows the same control application to be used either in a simulated virtual hardware or in real hardware.

\subsubsection{CoopDynSim}

The CoopDynSim simulator (figure 3) is developed in C++, renders objects using OpenGL and represents their physical counterpart using Newton Game Dynamics physics engine. Being developed towards mobile robots, the CoopDynSim had to be upgraded to simulate robotic manipulators, and to emulate the operating room environment. The operating room objects were designed from scratch using CAD softwares like Solidworks and Deep Exploration. On the other hand, the manipulator's 3D models were available at the producer website and were integrally added to the simulator keeping the dimensions and joint positions as close as possible to reality. It was also added a basic robotic base to account for the mobile platform and several end-effector instruments to would be handled by the manipulator during the procedure.

All the objects within the operating room were represented both graphically and physically and placed in the same positions as noted in the real environment. By doing so, we can assess the best position to place the robot during the surgery, to avoid crossing the neurosurgeon line of sight and colliding with the environment. The virtual robotic manipulator joints were implemented so that they could answer to target positions or velocity commands. By emulating these features from a real manipulator, it is possible to test not only the Geometric but also the developed Differential Kinematics.

\subsubsection{Middleware communication layer}

The middleware layer was implemented using YARP, an open-source software library oriented to robotic platforms, to establish communication protocols. This library brought the abstraction needed to define communication protocols

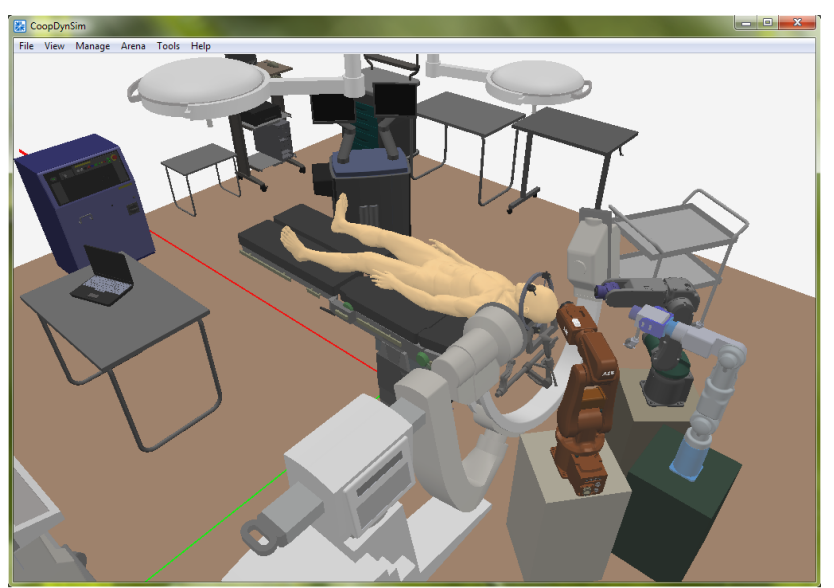

Figure 3. 3D simulator for robotic assisted DBS neurosurgery that allows emulating several hardware setups within the operating room, to test and assess their performance.

between applications developed in different languages, interfacing with hardware devices while supporting a wide range of connection types. In our project it provides a wrapper for the communication of each robot implemented APIs to the control application based in a socket interface. The robot's APIs are associated to a server port that connects to the control application's client port and communicate via a message protocol.

The communication message consists of a flexible structure that includes an error code, a string variable, a unique key command that specifies the type of message, a $\mathrm{M}$ number of integer parameters and a $\mathrm{N}$ number of floating point parameters.

\subsubsection{Control application}

The control application (figure 4) was implemented in $M A T L A B$, due to the inherent quick and ease algorithm testing, user interface implementation and also because it was the neurosurgeons' request. The control application establishes the communication between the server and client ports. The application includes information about each manipulator (Abb IRB 120, Motoman MH5 and Schunk Amtec LightWeightArm II) dimensions, joint limits and kinematic expressions.

The control application user interface is currently in a developing and testing stage. Saying that, the interface provides low level controls that allow the user to move each joint individually, to continuously read the end-effector position $^{2}$ and to set a desired position and orientation ${ }^{3}$ for the end-effector to reach. The Cartesian space variables for both position and orientation are handled with respect to the surgical referential rather than the world referential,

\footnotetext{
${ }^{2}$ Geometric Forward Kinematics

${ }^{3}$ Geometric Inverse Kinematics
} 


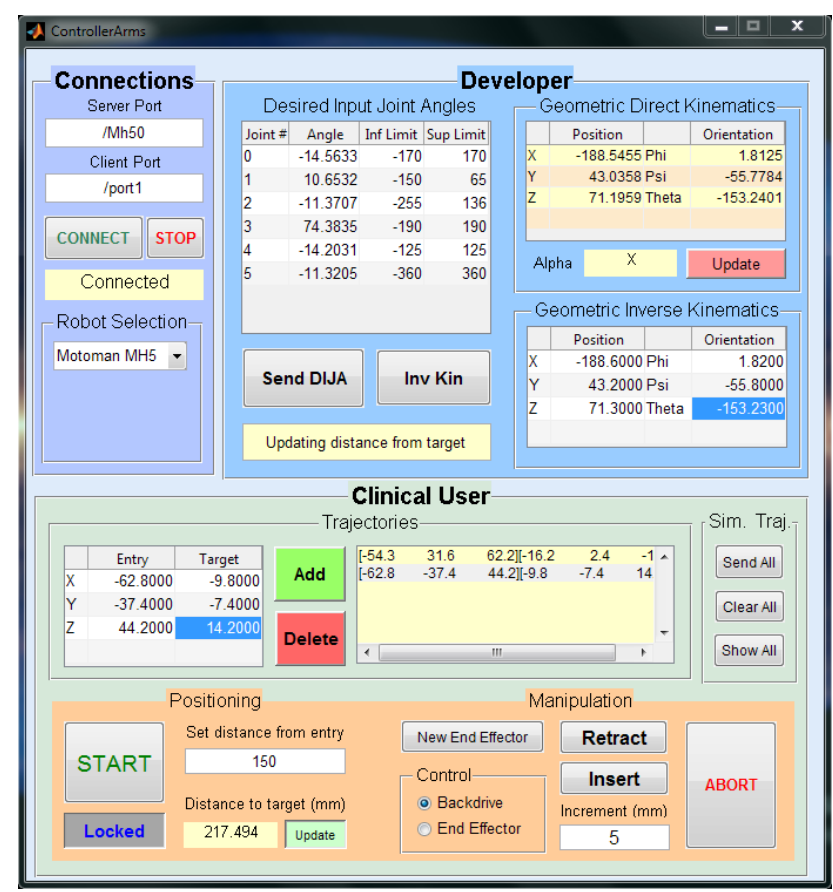

Figure 4. User interface of the control application for system developers and clinicians.

since the imaging planning software and all intra operative process is also managed relative to the surgical referential.

Since simulated robotic manipulators can handle different end-effectors, the control application recognizes which one is coupled to the arm. The available endeffectors, include static objects and 1 DOF slider actuators to linearly move instrumentation. Depending on the endeffector used, the control software applies transformations to kinematic expressions accordingly. The user knows at all times where is the instrumentation tip and how it is oriented, taking into account the 1 DOF slider displacement.

Considering that the imaging software provides the target location and the entry point, it was implemented a feature that allows the user to directly input these coordinates through the control application and visualize both the target and trajectory in a virtual world. It is possible to insert, remove and manage several trajectories at a time; the user can also select one trajectory to be followed by the robotic manipulator. When one trajectory is selected it remains highlighted in the simulated world, while the others stay hidden. The target is represented by a red sphere, while the trajectories are represented by a cylindrical green path. The diameters of both can be adjusted to assess the consistency of the executed movements within the desired trajectory. Such feature is very useful to debug the manipulator's performance.

Upon selecting a trajectory, the user can input a distance to the entry point. The manipulator places its endeffector collinear to the chosen trajectory, with its tip at the specified distance from the entry point. At this position, the user can move the end-effector along the trajectory either by Differential kinematics, or by controlling the $1 \mathrm{DOF}$ slider joint. It is given the choice to move the instrumentation inward or backward, for a specified distance in millimeters. The user may toggle a continuously updated display with the distance from the instrumentation tip to the target anatomical structure.

The application delivers status messages to the user notifying the success on delivering and receiving information. Both simulator and control application are enabled with safety mechanics that prevent any action when the communication fails. At the same time the control application has several fields to inform the user of how information is being handled and of any successful or failed routines. It was also implemented a safety subroutine that stops all the manipulator actions by pressing an emergency button.

\section{Conclusion}

These being the first steps of a long term project, a lot of information was already gathered through attending DBS surgeries and from the close cooperation of professionals in the area. In the end, we aim for a precision manipulation tool with further precision than the mechanical stereotactic frame without the need to follow the mount/dismount process for each trajectory specified. It should assist the neurosurgeon by holding and manipulating instrumentation and constraining its motions outside the surgical field, thus avoiding potential errors. Replacing a cumbersome mechanical device by a digital system enables the information to be managed by software, where verification routines can be used to consistently assess intraoperative data.

Robotic assisted DBS can improve the neurosurgeon's work experience and lead to better surgery outcomes as a result of the better precision and consistency on instrumentation handling. As an emergent technology only recently the first commercially available solutions were launched. However based on the information gathered so far we believe that it is possible to develop a pragmatic robotic system to assist neurosurgeons in stereotactic procedures within an affordable budget, thus democratizing the use of this tool.

As expected, there are also critical issues that need to be addressed in future work such as the need to devise a mobile platform to provide some portability to the robotic system and an attach system to fix the platform to the patient's reference stereotactic system. Another goal to achieve is the development of a system to recognize the robot's position and orientation relative to the surgical referential, as this transformation isn't static.

\section{Acknowledgements}

This work has been partially financed by projects FP7 Marie Curie ITN - NETT (project $\mathrm{n}^{\circ}$ 289146), 
FCT FCOMP-01-0124-FEDER-022674 and Pest-C/MATUI0013/2011 (FCT grant ref. UMINHO/BIC/8/2012).

\section{References}

[1] William J. Marks Jr., The expanding role of deep brain stimulation, in William J. Marks Jr.(Ed.), Deep Brain Stimulation Management (Cambridge University Press, 2010) 1-3.

[2] E. B. Montgomery Jr. and J. T. Gale, Mechanisms of action of deep brain stimulation (DBS), Neuroscience Biobehavioral Reviews, 32(3), 2008, 388-407.

[3] J. S. Perlmutter and J. W. Mink, Deep brain stimulation, Annual Review of Neuroscience, 29(1), 2006, 229-57.

[4] L. de Lau, Epidemiology of Parkinsons disease, The Lancet Neurology, 5(6), 2006, 525-535.

[5] E. Louis and R. Ottman, How common is the most common adult movement disorder? Estimates of the prevalence of essential tremor throughout the world, Movement disorders, 13(1), 1998, 5-10.

[6] A. Neligan and J. W. Sander, The incidence and prevalence of epilepsy, in International League Against Epilepsy's 13th, Epilepsy 2011, 2011, pp. 1-7.

[7] T. Wichmann and M. R. DeLong, Deep brain stimulation for neurologic and neuropsychiatric disorders, Neuron, 52(1), 2006, 197-204.

[8] F. Cardinale and R. Mai, Robotic Implantation of Intracerebral Electrodes in Epilepsy Surgery, Newsmagazine of The Congress of Neurosurgical Surgeons Spring, 12(2), 2011, 24-26.

[9] F. Seijo, M. Alvarez-Vega, B. Lozano, F. FernndezGonzlez, E. Santamarta and A. Saz, Common Questions and Answers to Deep Brain Stimulation Surgery, in M. H. Rogers and P. B. Anderson (Eds.), Deep Brain Stimulation: Applications, Complications And Side Effects (New York: Nova Science Publishers, 1st edition, 2009) 1-29.

[10] P. A. Starr, A. J. Martin, J. L. Ostrem, P. Talke, N. Levesque, and P. S. Larson, Subthalamic nucleus deep brain stimulator placement using high-field interventional magnetic resonance imaging and a skull-mounted aiming device: technique and application accuracy., Journal of Neurosurgery, 112(3), 2010, 479-490.

[11] H. B. Stewart, Surgical placement of deep brain stimulation leads for the treatment of movement disorders: intraoperative aspects, in W. J. Marks Jr. (Ed.), Deep Brain Stimulation Management (Cambridge University Press, 1st edition, 2010) 20-31.

[12] T. Haidegger, L. Kovacs, G. Fordos, Z. Benyo, P. Kazanzides, A. Katashev, Y. Dekhtyar, J. Spigulis, and R. Magjarevic, Future Trends in Robotic Neurosurgery, 14th Nordic-Baltic Conference on Biomedical Engineering and Medical Physics, Riga, Latvia, 2008, 229-233.

[13] K. Cleary and C. Nguyen, State of the art in surgical robotics: clinical applications and technology challenges., Computer aided surgery official journal of the International Society for Computer Aided Surgery, 6(6), 2001, 312-328.
[14] N. Nathoo, M. C. Cavusoglu, M. A. Vogelbaum, and G. H. Barnett, In touch with robotics: neurosurgery for the future., Neurosurgery, 56(3), 2005, 421-433.

[15] C. S. Karas and E. A. Chiocca, Neurosurgical robotics: a review of brain and spine applications, Journal of Robotic Surgery, 1(1), 2007, 39-43.

[16] M. Zimmermann, R. Krishnan, A. Raabe, and V. Seifert, Robot-assisted navigated endoscopic ventriculostomy: implementation of a new technology and first clinical results., Acta Neurochirurgica, 146(7), 2004, 697704.

[17] N. Villotte, D. Glauser, P. Flury, and C. W. Burckhardt, Conception of stereotactic instruments for the neurosurgical robot Minerva, 14th Annual International Conference of the IEEE Engineering in Medicine and Biology Society $E M B C$, Paris, France, 1992, 1089-1090.

[18] G. R. Sutherland, P. B. McBeth, and D. F. Louw, NeuroArm: an MR compatible robot for microsurgery, International Congress Series, vol. 1256, 2003, 504-508.

[19] B. Davies, S. Starkie, S. Harris, E. Agterhuis, V. Paul, and L. M. Auer, Neurobot: a special-purpose robot for neurosurgery, Proceedings 2000 ICRA IEEE International Conference on Robotics and Automation, San Francisco, California, 2000, 4103-4108.

[20] Q. H. Li, L. Zamorano, A. Pandya, R. Perez, J. Gong, and F. Diaz, The application accuracy of the NeuroMate robot-A quantitative comparison with frameless and frame-based surgical localization systems., Computer aided surgery official journal of the International Society for Computer Aided Surgery, 7(2), 2002, 90-98.

[21] M. S. Eljamel, Validation of the PathFinder neurosurgical robot using a phantom., The international journal of medical robotics computer assisted surgery MRCAS, 3(4), 2007, 372-377.

[22] M. D. Comparetti, A. Vaccarella, D. D. Lorenzo, G. Ferrigno, and E. D. Momi, Multi-robotic approach for keyhole neurosurgery: the ROBOCAST project, SCATh Joint Workshop on New Technologies for Computer/Robot Assisted Surgery, Graz, Austria, 2011, 1-4.

[23] Medtech S.A, Premarket Notification 510(K) Submission Rosa Surgical Device. Montpellier, France, 2010, 610.

[24] B. L. Davies, S. J. Harris, W. J. Lin, R. D. Hibberd, R. Middleton, and J. C. Cobb, Active compliance in robotic surgery - the use of force control as a dynamic constraint, Journal of Engineering in Medicine, vol. 211 Part H, 1997, 285-292.

[25] C. Faria, Robotic implantation of intracerebral electrodes for deep brain stimulation, M.Sc. Dissertation, University of Minho, 2012.

[26] T. Machado, M. Sousa, S. Monteiro, and E. Bicho, CoopDynSim: a 3D robotics simulator, Robotica, Guimaraes, Portugal, 2012, 45-50. 